derived, for it is hard to arrive at any other result by any first-order principle. The result of the experiment is equally gratifying to the relativist and to the classicist. It was pointed out to the writer by $\mathrm{K}$. T. Compton that this uncertainty of 3 per cent. might cover the failure to detect the 5 per cent. slip ( 20 out of $200 \mathrm{~km}$. $/ \mathrm{sec}$. or more) which Miller's result might suggest.

As Watson described this heroic experiment, a feeling of disappointment arose that such glorious apparatus had to be dismantled. The pipes ( 14 in.) were well and truly laid over a large area, and it was necessary to make good joints to get a vacuum, but winter was coming on and frost would have displaced the pipes, so that it is a greater matter for congratulation that this fine experiment should have been carried out, than that the apparatus should have been dismantled and removed.

It is possible to summarise the situation:

\section{For Relativity.}

Mercury perihelion (?). Eclipse results. Spectrum shift.

\author{
Against Relativity, \\ but for drag and slip. \\ Miller's experiments \\ on \\ Mount Wilson.
}

\section{For Relativity or for Stagnant Ether, but against drag and slip.}

Michelson-Gale-Silberstein. Chicago rotation experiment.

This brief and imperfect record of a difficult subject is written for those who are interested in the situation as it appeared to one who attended the meetings at Kansas City.

\title{
Some Properties of the Vitamins.
}

$\mathrm{T}^{\mathrm{H}}$ $\mathrm{HE}$ reality of the existence of the three well-known vitamins having been confirmed by numerous careful investigations, attention was directed to their isolation in a pure state, with the ultimate aim of discovering their chemical constitution. At first the work was largely qualitative, but with improvement in the technique of the necessary animal experiments, quantitative investigations became possible, and have led not only to a great increase in our knowledge of these elusive substances, but also to the probable discovery of further members of the series. Whilst undue multiplication of the number of unknown accessory food factors must be deprecated, yet it must be admitted that recent work suggests that there exist besides the orthodox trinity, an antirachitic factor $D$ and an antisterility vitamin $\mathrm{E}$ : at the same time, our knowledge of their chemical properties has advanced so considerably, especially in the case of the fat-soluble vitamins $\mathrm{A}$ and $\mathrm{D}$, that some of them may soon be justifiably transferred to a place amongst compounds of a similar chemical constitution. Thus the multiplication of vitamins resolves itself into the problem of the identification of substances which are required in metabolism only in minute amounts, and the discovery of which has been dclayed until recently owing to difficulties of chemical analysis, and to the fact that they are usually present in the natural food of animals in adequate amount. Similar problems can be found in other branches of biochemistry and metabolism : thus all the amino-acids which make up the protein molecule have not yet probably been isolated, and vitamins are not the only substances which are required in minute quantities-for example, certain elements such as iodine.

Recent investigation has made it clear that the fatsoluble vitamins are present in the unsaponifiable portion of the fat, and that cholesterol can be removed from this fraction without impairing its efficacy. Takahashi and his co-workers have now carried our knowledge a stage further in an elaborate investigation (K. Takahashi, Z. Nakamiya, K. Kawakami, and T. Kitasato, Scient. Papers, Inst. Phys, and Chem. Res., Tokyo, I925, vol. 3, p. 8I). Starting with cod-liver oil or green leaves, the unsaponifiable fraction was obtained in a fairly pure condition and the cholesterol removed; some further purification of the product isolated from the former source was attempted by crystallisation from acetone at $-60^{\circ}$ to $-70^{\circ} \mathrm{C}$., followed by distillation at $147^{\circ}-150^{\circ} \mathrm{C}$. under $0.02-0.03 \mathrm{~mm}$. pressure. These drastic treatments did not appear to destroy the vitamin, but did not effect any great purification : the products obtained from the two sources are somewhat different in composition, that from the oil being the more unsaturated. The yields of the crude material were of the order of $O^{\circ} I$ per cent of the oil and 0.025 per cent of the dried leaves. Tested on animals (rats), about $0.04 \mathrm{mgm}$. of the crude material or $0.005 \mathrm{mgm}$. of the purified product sufficed, when given daily to each animal, to promote growth on a diet deficient only in vitamin $\mathrm{A}$.

The Japanese investigators have made a lengthy investigation into the physical and chemical properties of their refined product, which they have labelled 'Biosterin.' It has an absorption band in the ultraviolet, it is soluble in the organic solvents, but is unstable in ether and chloroform, and it is adsorbed by fuller's earth and animal charcoal. It also produces a photo-chemical action upon photographic plates, which the authors suggest, as the result of a series of experiments, is due to the formation of active oxygen from the oxygen of the air over the material: a similar effect was found to be produced, though less markedly, by the cod-liver oil itself, and also, but very feebly, by olive oil. As mentioned above, the vitamin is also stable to heat, in the absence of air. O. Rosenheim and J. C. Drummond (Biochem. Journ., I925, vol. I9, p. 753) have also shown that the cholesterol free unsaponifiable fraction of cod-liver oil containing the vitamin can be distilled in superheated steam in an atmosphere of nitrogen or in a high vacuum without loss of activity. On the other hand, H. W. Southgate (ibid., p. 733) has found that when the cod-liver oil itself is heated to $200^{\circ} \mathrm{C}$. in an atmosphere of carbon dioxide, and maintained at this temperature for varying lengths of time, a slow destruction of both the growthpromoting and the antirachitic factors takes place: the author was unable to show that this destruction was due to the liberation of fatty acids by hydrolysis at the high temperature used.

The stability to heat of these vitamins is of considerable practical importance: thus they are likely to be

$$
\text { NO. 2945, VOL. I I } 7 \text { ] }
$$


found in certain foods, such as milk, which has been dried by means of heat. Gladys A. Hartwell (Brit. Med. Journ., I925, vol. I, p. 1073) has shown that both dried and evaporated milks form, together with white bread, diets adequate for growth and reproduction in rats: the former was as good as, or better than, fresh cow's milk, whilst the latter was distinctly inferior, this inferiority showing itself more especially when the animals reached adult size. It is possible that this difference is due to a relative deficiency of vitamin $B$ in the evaporated milk. It must be pointed out that these results do not imply an adequate supply of vitamin C, since the rat requires extremely little of this vitamin compared to the guinea-pig or man: moreover, vitamin $\mathrm{C}$ is the most sensitive to the ordinary destructive agents. Katharine $\mathrm{H}$. Coward (Biochem. Journ., 1925 , vol. I9, p. 500) has found also that drying green leaves need not destroy vitamin $\mathrm{A}$, though it appears to vanish in leaves which are allowed to wither. The importance of the vitamin content of milk appears again in some recent experiments by H. Pringle ( $\mathrm{Sci}$. Proc. Roy. Dub. Soc., I925, vol. x8, p. 93). Human milk was found to be less efficient in promoting the growth of young rats than cow's milk, probably owing to a deficiency in vitamin $B$ content. Thus again is the importance of the mother's diet during pregnancy and lactation emphasised.

Takahashi and his co-workers have carried out a number of analyses of the purified products obtained from cod-liver oil and green leaves, and suggest that their formulæ should be $\mathrm{C}_{27} \mathrm{H}_{44} \mathrm{O}_{2}$ and $\mathrm{C}_{27} \mathrm{H}_{46} \mathrm{O}_{2}$ respectively: for comparison, that of cholesterol, $\mathrm{C}_{27} \mathrm{H}_{46} \mathrm{O}$, may also be given. A large series of chemical derivatives have been prepared, from which the authors have deduced that the oxygen atoms are present as $\mathrm{OH}$ groups, one a tertiary, and the other a primary or secondary alcohol, and that the products are unsaturated, containing three double bonds : they are also capable of reduction, and form a digitonide but less readily than cholesterol : on standing they slowly take up oxygen. In general, it may be stated that any change in the molecule results in a loss of the growth-promoting property. Cholesterol and its oxy derivative, which has the same formula as the product obtained from green leaves, are also inactive.

At the present time the most useful chemical property of vitamin $\mathrm{A}$ is probably that of producing a blue colour in the presence of certain reagents. Takahashi has investigated this side of the subject, using a number of different reagents, such as sulphuric acid and fuller's earth: inactive allied products give a reddish colour under similar conditions. Rosenheim and Drummond have utilised arsenic chloride, trichloracetic acid, dimethylsulphate, etc., for a similar purpose, and have found that the intensity of the blue colour runs parallel with the product's potency when tested on animals, in the case of both butter and cod-liver oil. Incidentally it may be mentioned that the antirachitic factor, in the form of cholesterol activated by exposure to ultraviolet light, does not give any colour with these reagents, a further confirmation of the distinctness of vitamins A and $D$. The test is very delicate, the cholesterol free unsaponifiable fraction giving it in a dilution of one in two million. The colour is discharged by a number of substances, including alcohol and ether.
One point may be referred to before leaving the work of the Japanese investigators, and that is that they have found their products toxic to rats in doses of about so mgm. a day. The possibility that this might be due to some poisonous impurity, perhaps introduced during the requisite chemical manipulations, does not appear to have been considered. It is obvious that the value of their conclusions will be considerably enhanced when the work has been repeated-and confirmed-by independent observers.

The relation of light to the fat-soluble vitamins has not yet been completely elucidated, but it appears from the evidence available that it produces synthesis of both the growth-promoting and antirachitic factors in plants, but in animals only influences the latter. Plants grown in the dark produce no vitamin $\mathrm{A}$, but even one day's exposure to light is sufficient to cause its appearance: at the same time the lipochrome pigments are developed (K. H. Coward, ibid.). The relation of ultra-violet light to the antirachitic factor in plants has been little studied, since both factors usually appear to occur together: in animals, light does not cause any synthesis of vitamin A, but may influence the production of the antirachitic factor, for example, by activating the cholesterol in the skin. Whether this is the case or not, the improvement in calcification in rickets is probably in part due to an increased calcium absorption; since in other conditions in which there is a drain on the calcium of the body, for example, in lactation, exposure of the animal to ultra-violet light results in increased calcium absorption and retention (see the experiments on goats by J. B. Orr, H. E. Magee, and J. M. Henderson, Biochem. Journ., 1925, vol. 19, p. 569).

A further factor to be considered in this increased retention of calcium is the influence of small quantities of iodine in the diet. F. C. Kelly (ibid., p. 559) has shown that this addition to the diet of the growing pig produces an increased retention of nitrogen, phosphorus, and calcium, and points out that cod-liver oil is a rich source of this element. Iodine is necessary for the production of the thyroid hormone, which is itself essential for growth, but the relation, if any, between this secretion and the growth vitamins is at present unknown.

Whether light has any influence on the other vitamins is at present doubtful. In fact, so far as vitamin C is concerned, P. Eggleton and L. T. Harris (Brit. Med. Journ., r925, vol. 2, p. 989) have recently shown that oats germinated in the dark produce the vitamin in proportion to the amount of growth occurring ; moreover, ultra-violet light cannot prevent or cure experimental scurvy in guinea-pigs on a scorbutic diet. Hence it cannot replace an adequate supply of this vitamin in the food.

Less is known of the chemical nature of the watersoluble vitamins than of that of the fat-soluble. S. S. Zilva (Biochem. Journ., I925, vol. I9, p. 589) has obtained vitamin $\mathrm{C}$ in a concentrated condition from orange and swede juice. The nitrogen content of this fraction was only I-2 mgm. per cent., of which about half was amino and amide nitrogen; the latter could be destroyed without interference with the potency of the preparation. R. A. Peters (ibid., r924, vol. r8, p. 858 ) has obtained a somewhat similar result with a 
concentrated solution of vitamin $B$ extracted from yeast; nitrous acid does not destroy it, as is the case with primary and many secondary amines. Working with $\mathrm{H}$. W. Kinnersley, he has succeeded in purifying the yeast extract still further, until only $0.084 \mathrm{mgm}$, is necessary to cure symptoms of polyneuritis in a pigeon (ibid., 1925, vol. 19, p. 820). Regular doses given to a bird on a polished rice diet are capable of preventing the appearance of symptoms without, however, maintaining its weight. Now vitamin B is essential for growth and maintenance of weight in rats, so that the authors are led by their results to suggest that the factor which cures and prevents polyneuritis in pigeons may be different from the one which is necessary for growth and maintenance of weight. It is possible, however, that the continued loss of weight without symptoms may be due to the deficiency of the polished rice dict in some other material.

It has been known for some time that excessive amounts of carbohydrate food hasten the onset of symptoms in pigeons. In examining the relationship between vitamin $B$ and carbohydrate metabolism, $\mathrm{P}$. Eggleton and L. Gross (ibid., I925, vol. I9, p. 633) have found that deprivation of vitamin $B$ in rats leads in about a month to a fall in the blood-sugar, together with a gradual disappearance of glycogen from the liver ; at the same time Kinnersley and Peters noticed that the convulsions of polyneuritic pigeons occurred at low temperatures, whilst removal of the birds to the warmth of the laboratory produced a temporary improvement. Now it is known that in mice the convulsions produced by insulin only occur regularly if the animals are kept at an external temperature so high as $37^{\circ} \mathrm{C}$. At first sight the two observations appear opposed; but the condition of the experimental animals is fundamentally different: the mice have glycogen in their livers, whilst the pigeons probably do not. Hence cold in the former, stimulating glycogenolysis, acts against the insulin injected by producing a flow of sugar into the blood and thus prevents hypoglycæmic symptoms, whilst in the latter this cannot occur owing to the paucity of glycogen in the liver. $\Lambda$ the same time, the cold increases the metabolism of the muscles, leading to withdrawal of sugar from the blood, which cannot be replaced, thus producing convulsions: warmth would diminish the demand for sugar and allow the blood-sugar to be restored to a more normal level. Some such hypothesis may be useful in correlating the various observations which have been recorded.

In conclusion, attention may be directed to some recent work in connexion with vitamin $\mathrm{E}$, first described by Evans and Bishop a few years ago. It was observed that rats on certain synthetic diets, chiefly those in which the protein was supplied by casein, became sterile in course of time: any offspring of these rats obtained before sterility occurred were sterile from birth. In the female the ovaries are normal, the sterility being shown by absorption of the embryos late in gestation. In the male, on the other hand, the sterility is produced by degeneration of the testes themselves : a full description of the histological changes involved has been given by K. E. Mason (Proc. Nat. Acad. Sci., U.S.A., 1925, vol. rx, p. 377). Degeneration begins about two months after the commencement of the diet, and recovery only occurs when the vitamin is given if some normal testicular tissue is still in existence.

The distribution of the vitamin $\mathrm{E}$ and its properties are considered in more detail by H. M. Evans and G. O. Burr (ibid., I925, vol. I I, pp. 334 and 378 ). It is fat soluble, and its richest source is the oil cxpressed from the germ of wheat: it occurs also in oats, corn, lettuce, alfalfa, etc. ; in small amounts in animal tissues including milk, and also in moderate amount in the vegetable oils. Cod-liver oil contains little. It is very stable and can be distilled in superheated steam; it occurs in the unsaponifiable fraction of the oil, but is not precipitated by digitonin: it does not contain nitrogen, sulphur, phosphorus, or the halogens. A single dose of $5 \mathrm{mgm}$. of the concentrated product by mouth or subcutaneously to a female rat suffices for the production of a normal litter, whilst $0.3 \mathrm{mgm}$. daily prevents male sterility. It appears to be stored in the body to a considerable extent, but is used up in the ordinary processes of metabolism quite apart from pregnancy. In some of its properties, therefore, it resembles the other fat soluble vitamins, but in its distribution, and in the fact that normal growth occurs in its absence, it is sharply differentiated from them.

\section{News and Views.}

AT a conference on March 25, held by the principal broadcasting organisations, representatives from twenty European countries took part. A new scheme for the redistribution of wave-lengths in Europe was submitted and provisionally approved. The details of the scheme will now be considered by the various interested organisations from the point of view of local difficulties. Suggested amendments will be considered by the Committee of the Office International of Radiophonie, and a final scheme will be drawn up for the approval of the various Governments concerned. The scheme divides broadcasting stations into two classes. The first class consists of those capable of guaranteeing good reception at long ranges. They receive an 'exclusive' wave-length, but every country receives at least one exclusive wave-length. The other class consists of low-power stations for the use of local listeners only. All this class will work on a common wave-length. Experiments have shown that each of these small stations can work without practical interference from the other stations using the same wave-length. To ensure satisfactory results it is absolutely necessary that the same standard type of wave-metre should be used by all the stations. This problem is being considered by a technical committee at Brussels under M. Raymond Braillard. No wave-lengths less than 200 metres have been allotted, and great pains have becn taken to avoid unnecessary changes in wave-lengths already in use. It will be seen that the proposed solution is, perhaps unavoidably, not a perfect one. The ideal solution would be one in which every listener with a good valve set would be able to listen in to many of the small stations in Europe. If they all have a common

$$
\text { NO. 2945, VOL. I I } 7 \text { ] }
$$

